\title{
OSCILLATION RESULTS FOR SOME LINEAR DIFFERENTIAL EQUATIONS
}

\author{
YIK-MAN CHIANG, ILPO LAINE and SHUPEI WANG
}

\section{Introduction.}

The zero distribution of solutions of linear differential equations of type,

$$
f^{\prime \prime}+A(z) f=0,
$$

where $A(z)$ is a transcendental entire function, has been actively investigated during the last decade, see [2] for the starting point of these investigations. The exponent of convergence $\lambda(f)$ of the zero-sequence of $f$ has been the standard device to measure the frequency of the zeros. The major conjecture, still remaining open, is that whenever the order $\sigma(A)$ of $A$ is finite and not an integer, then $\max \left(\lambda\left(f_{1}\right), \lambda\left(f_{2}\right)\right)=+\infty$, provided $f_{1}, f_{2}$ are linearly independent solutions of (1.1). Most of the research work dealing (1.1) during the last decade has been written towards proving this conjecture, at least partially. A special case, frequently studied, is given by equations of type,

$$
f^{\prime \prime}+\left(R e^{P}+Q\right) f=0,
$$

where $R, P, Q$ are polynomials, see [4], [5], [6], [7], [15] and [16]. The starting point of this paper is the following typical " $\frac{1}{16}$-theorem", see [5], Theorem 1:

Theorem A. Let $P$ be a polynomial of degree $\operatorname{deg} P=n \geqq 1$, and let $Q$ be an entire function of order $\sigma(Q)<n$. Suppose that

$$
f^{\prime \prime}+\left(e^{P}+Q\right) f=0
$$

admits a non-trivial solution $f$ such that $\lambda(f)<n$. Then $f$ has no zeros, $Q$ is a polynomial and

$$
Q=-\frac{1}{16}\left(P^{\prime}\right)^{2}+\frac{1}{4} P^{\prime \prime}
$$

Supported in part by the Finnish Academy grants 1021097 and 2592, by the University of Joensuu, by the Hong Kong University of Science and Technology grant DAG92/93.SC22 and by the Center for International Mobility in Finland.

Received May 20, 1994. 
This paper consists of three parts. We first prove some results directly related to Theorem A. Secondly, we give some similar results for solutions to corresponding linear differential equations of higher order. Finally, we consider periodic linear differential equations. In what follows, we assume that the reader is familiar with the basic notions and results of the Nevanlinna theory, see e.g. [12], [13] and [14]. In particular, $S(r, f)$ means any quantity of growth $o(T(r, f))$ as $r \rightarrow \infty$ outside of a possible exceptional set of finite linear measure.

\section{The second order case.}

In Theorem $\mathrm{A}$, it remains an open question whether $n \leqq \lambda(f)<\infty$ may happen for a non-trivial solution $f$ of (1.3). We give a negative partial solution to this question by proving

THEOREM 2.1. Let $P(z)$ be a polynomial of degree $n \geqq 1$, and $Q(z)$ be a transcendental entire function of order $\sigma(Q)<n$. Then any non-trivial solution $f$ of $(1.3)$ satisfies $\lambda(f)=\infty$.

Of course, Theorem A and Theorem 2.1 address the case when the coefficient in (1.1) is of finite order. Our second result treats a special case of $(1.1)$ where this coefficient is of infinite order. Theorem 2.2 may be considered an analogue.

THEOREM 2.2. Let $B(z)$ be a transcendental entire function, and let $A(z) \neq 0$, and $C(z)$ be entire functions of finite order. Then for any non-trivial solution $f$ to

$$
f^{\prime \prime}+\left(A(z) e^{B(z)}+C(z)\right) f=0,
$$

we have either $\lambda(f)=\infty$, or $f$ admits no zeros, $A(z)$ has no zeros, and

$$
C=-\frac{1}{16}\left(\frac{A^{\prime}}{A}+B^{\prime}\right)^{2}+\frac{1}{4}\left(\frac{A^{\prime}}{A}+B^{\prime}\right)^{\prime} .
$$

REMARK. In the special case of $C(z) \equiv 0,(2.2)$ reduces to

$$
B^{\prime 2}=4 B^{\prime \prime}-2 \frac{A^{\prime}}{A} B^{\prime}+4\left(\frac{A^{\prime}}{A}\right)^{\prime}-\left(\frac{A^{\prime}}{A}\right)^{2} .
$$

By the standard Clunie-type argument, see [14], Lemma 2.4.2, we deduce from (2.3) that $m\left(r, B^{\prime}\right)=O(\log r)$. Hence, $B^{\prime}$ is a polynomial, contradicting the above assumption. Therefore, in the case of $C(z) \equiv 0$, any non-trivial solution of (2.1) must satisfy $\lambda(f)=\infty$, see [2], Theorem 2(B), where such a result is proved.

To prove Theorem 2.1 and Theorem 2.2, we have to recall some known lemmas. Lemma 2.1 is nothing but an easy variant of the standard Clunie lemma [10], Lemma 1. 
LEMMA 2.1. Let $f$ be a non-constant meromorphic function, and let $P\left(z, f, f^{\prime}, \ldots, f^{(k)}\right), Q\left(z, f, f^{\prime}, \ldots, f^{(k)}\right)$ be two differential polynomials of $f$, not identically zero, with the total degree of $Q$ being $\leqq n$. If

$$
f^{n} P\left(z, f, f^{\prime}, \ldots, f^{(k)}\right)=Q\left(z, f, f^{\prime}, \ldots, f^{(k)}\right),
$$

then

$$
m(r, P) \leqq S(r, f)+\sum_{\alpha(z)} m(r, \alpha(z))
$$

where the sum extends over all coefficients of $P$ and $Q$.

Next, write $P(z)$ in (1.3) in the form

$$
P(z)=(\alpha+i \beta) z^{n}+\ldots+a_{0}, \quad \alpha+i \beta \neq 0, \quad n \geqq 1, \quad \alpha, \beta \text { real, }
$$

and define

$$
\delta(P, \theta):=\alpha \cos n \theta-\beta \sin n \theta .
$$

Then $\delta(P, \theta)=0$ on the rays

$$
\arg z=\theta_{j}:=\tilde{\theta}+\frac{j}{n} \pi, \quad j=0,1, \ldots, 2 n-1,
$$

which form $2 n$ sectors of opening $\pi / n$ for some $\tilde{\theta}$. We denote by $S_{1}^{+}, \ldots, S_{n}^{+}$(resp. $S_{1}^{-}, \ldots, S_{n}^{-}$) those open sectors where $\delta(P, \theta)>0$ (resp. $\delta(P, \theta)<0$ ). We also denote $S^{+}=\bigcup_{i=1}^{n} S_{i}^{+}, S^{-}=\bigcup_{i=1}^{n} S_{i}^{-}$. With this notation, it is easy to deduce the following lemma, see [6], Lemma 3, and [14], Lemma 5.14.

LeMma 2.2. Let $P(z)$ be a polynomial of degree $n \geqq 1$, and let $\varepsilon>0$ be a given constant. Then we have:

(1) If $\delta(P, \theta)>0$, there exists an $r(\theta)>0$ such that for any $r \geqq r(\theta)$,

$$
\left|e^{P\left(r e^{i \theta}\right)}\right| \geqq \exp \left((1-\varepsilon) \delta(P, \theta) r^{n}\right) .
$$

(2) If $\delta(P, \theta)<0$, there exists an $r(\theta)>0$ such that for any $r \geqq r(\theta)$,

$$
\left|e^{P\left(r e^{i \theta}\right)}\right| \leqq \exp \left((1-\varepsilon) \delta(P, \theta) r^{n}\right) .
$$

Lemma 2.3 below is just a special case of [6], Lemma 2, see [14], Lemma 5.15.

LEMMA 2.3. Let $P(z), S(z)$ be two polynomials and let $\theta_{0}$ be such that $\delta\left(P, \theta_{0}\right)=$ 0. Consider

$$
H(z):=e^{\frac{1}{2} P(z)} \int_{a}^{z} S(t) e^{-\frac{1}{2} P(t)} d t
$$


where $a \in \mathrm{C}$ is fixed. Then there exists a rational function $\tilde{Q}(z)$ such that for any $\theta \neq \theta_{0}$ we have

$$
H\left(r e^{i \theta}\right)=\tilde{Q}\left(r e^{i \theta}\right)+c(\theta) \cdot e^{\frac{1}{2} P\left(r e^{i \theta}\right)}+O\left(r^{-2}\right),
$$

as $r \rightarrow \infty$, where $c(\theta)$ is a constant, provided $\delta(P, \theta)>0$, while

$$
H\left(r e^{i \theta}\right)=\tilde{Q}\left(r e^{i \theta}\right)+O\left(r^{-1}\right)
$$

holds as $r \rightarrow \infty$, if $\delta(P, \theta)<0$.

Finally, we recall [11], Corollary 1:

LEMMA 2.4. Let $f$ be a meromorphic function of finite order $\rho$, let $\varepsilon>0$ be a given constant and let $k>j \geqq 0$ be integers. Then there exists a set $E_{0} \subset[0,2 \pi)$ of linear measure zero, such that if $\theta_{0} \in[0,2 \pi) \backslash E_{0}$, then there is a constant $R_{0}=R_{0}\left(\theta_{0}\right)>1$ such that

$$
\left|\frac{f^{(k)}\left(r e^{i \theta_{0}}\right)}{f^{(j)}\left(r e^{i \theta_{0}}\right)}\right| \leqq r^{(k-j)(\rho-1+\varepsilon)}
$$

for all $r \geqq R_{0}$.

2.1. Proof of Theorem 2.1. In this section, we always denote by $E_{0}$ a set in $[0,2 \pi)$ of linear measure zero, by $K$ and $N$ positive constants, by $\theta$ a given value in $[0,2 \pi)$, and by $R(\theta)$ a positive number (usually sufficiently large) depending on $\theta$. Each of these symbols will not necessarily be the same in different occurrences.

Suppose now that the equation (1.3) admits a non-trivial solution $f_{0}$ such that $\lambda\left(f_{0}\right)<\infty$. Write $f_{0}$ in the form

$$
f_{0}=\pi e^{h},
$$

where $h(z)$ is transcendental entire and $\pi(z)$ is the canonical product formed with the zeros of $f_{0}$; hence $\sigma(\pi)=\lambda\left(f_{0}\right)<\infty$. Substituting (2.4) into the equation (1.3) we obtain

$$
\left(h^{\prime}\right)^{2}=-h^{\prime \prime}-2 \frac{\pi^{\prime}}{\pi} h^{\prime}-\frac{\pi^{\prime \prime}}{\pi}-e^{P}-Q .
$$

By Lemma 2.1, (2.5) implies that $\sigma\left(h^{\prime}\right)<\infty$. Furthermore, differentiation of (2.5) and elimination of $e^{P}$ from (2.5) and the differentiated equation results in

$$
\begin{aligned}
2 S h^{\prime}= & -h^{\prime \prime \prime}+\left(P^{\prime}-2 \frac{\pi^{\prime}}{\pi}\right) h^{\prime \prime}+2\left(P^{\prime} \frac{\pi^{\prime}}{\pi}-\left(\frac{\pi^{\prime}}{\pi}\right)^{\prime}\right) h^{\prime} \\
& +P^{\prime} \frac{\pi^{\prime \prime}}{\pi}-\left(\frac{\pi^{\prime \prime}}{\pi}\right)^{\prime}+P^{\prime} Q-Q^{\prime},
\end{aligned}
$$


where

$$
S(z):=h^{\prime \prime}-\frac{1}{2} P^{\prime} h^{\prime}
$$

Again by Lemma 2.1, we get

$$
m(r, S) \leqq O\left(r^{\sigma_{1}}\right)
$$

for some $\sigma_{1}<n=\operatorname{deg} P$. Hence, $S(z)$ is an entire function of order $<n$. In fact, $S(z)$ reduces to a polynomial. This follows easily by proving that $S(z)$ is of polynomial growth along any ray $z=r e^{i \theta}$ in $S^{+} \backslash E_{0}$. In fact, once this has been established, then a standard Phragmén-Lindelöf argument implies that $S(z)$ has to be a polynomial. Indeed, around each of the negative sectors $S_{i}^{-}$, we may take two rays from the neighbouring sectors of $S_{i}^{-}$such that $S(z)$ is of polynomial growth on these two rays and that the opening between these two rays is less than $\pi / \sigma_{2}$ for some $\sigma_{2}$ such that $\sigma(S)<\sigma_{2}<n$. The Phragmén-Lindelöf principle now shows that $S(z)$ is of polynomial growth along any ray in $S_{i}^{-}$. Hence, $S(z)$ has to be a polynomial.

Consider now a fixed point on any ray $z=r e^{i \theta}$ in $S^{+} \backslash E_{0}$, where $E_{0} \subset[0,2 \pi)$ is a set of linear measure zero such that Lemma 2.4 may be applied whenever needed. If $\left|h^{\prime}\left(r e^{i \theta}\right)\right| \leqq 1$, then (2.7) and Lemma 2.4 imply that

$$
|S| \leqq\left|h^{\prime \prime}\right|+\frac{1}{2}\left|P^{\prime}\right| \leqq\left|\frac{h^{\prime \prime}}{h^{\prime}}\right|+\frac{1}{2}\left|P^{\prime}\right| \leqq K \cdot r^{N}
$$

holds along the ray $z=r e^{i \theta}$ for $r \geqq R(\theta)$ and for some $N$. On the other hand, if $\left|h^{\prime}\left(r e^{i \theta}\right)\right|>1$, then we have at $z=r e^{i \theta}$, by Lemma 2.4 and (2.6) that

$$
\begin{aligned}
|S| \leqq & \frac{1}{2}\left|\frac{h^{\prime \prime \prime}}{h^{\prime}}\right|+\frac{1}{2}\left|\frac{h^{\prime \prime}}{h^{\prime}}\right|\left(\left|P^{\prime}\right|+2\left|\frac{\pi^{\prime}}{\pi}\right|\right)+\left(\left|P^{\prime}\right|\left|\frac{\pi^{\prime}}{\pi}\right|+\left|\left(\frac{\pi^{\prime}}{\pi}\right)^{\prime}\right|\right) \\
& +\frac{1}{2\left|h^{\prime}\right|}\left(\left|P^{\prime}\right|\left|\frac{\pi^{\prime \prime}}{\pi}\right|+\left|\left(\frac{\pi^{\prime \prime}}{\pi}\right)^{\prime}\right|+\left|P^{\prime}\right||Q|+\left|Q^{\prime}\right|\right) \\
& \leqq K r^{N}+\frac{e^{r^{\sigma_{3}}}}{\left|h^{\prime}\right|}
\end{aligned}
$$

for $r \geqq R(\theta)$ and for some $0<\sigma_{3}<n$, since $\sigma(Q)<n$. However, by (2.5) and the fact that $\left|h^{\prime}\left(r e^{i \theta}\right)\right|>1$, we get at $z=r e^{i \theta}$,

$$
\begin{aligned}
\left|e^{P}\right| & \leqq\left|h^{\prime}\right|^{2}+\left|h^{\prime \prime}\right|+2\left|h^{\prime}\right|\left|\frac{\pi^{\prime}}{\pi}\right|+\left|\frac{\pi^{\prime \prime}}{\pi}\right|+|Q| \\
& \leqq\left|h^{\prime}\right|^{2}\left(1+\left|\frac{h^{\prime \prime}}{h^{\prime}}\right|+2\left|\frac{\pi^{\prime}}{\pi}\right|\right)+\left|\frac{\pi^{\prime \prime}}{\pi}\right|+|Q| \\
& \leqq\left|h^{\prime}\right|^{2} \cdot r^{N}+e^{r^{\sigma_{4}}}
\end{aligned}
$$


for $r \geqq R(\theta)$ and for some $0<\sigma_{4}<n$, since $\sigma(Q)<n$. Hence, by Lemma 2.2, we can deduce from $(2.10)$ that

$$
\left|h^{\prime}\left(r e^{i \theta}\right)\right| \geqq e^{\frac{1}{8} \delta(P, \theta) r^{n}}
$$

for $r \geqq R(\theta)$. Therefore, by (2.11) and (2.9) we get

$$
\left|S\left(r e^{i \theta}\right)\right| \leqq K r^{N}
$$

for $r \geqq R(\theta)$, provided that $\left|h^{\prime}\left(r e^{i \theta}\right)\right|>1$.

By (2.8) and (2.12), we see that $S(z)$ is of polynomial growth along any ray in $S^{+} \backslash E_{0}$.

Next, we proceed to show that $Q(z)$ is of rational growth along any ray $\Gamma \in S^{-} \backslash E_{0}$, where $E_{0}$ again meets the hypothesis of Lemma 2.4. To this end, we solve the equation (2.7) for $h^{\prime}$ to get

$$
h^{\prime}(z)=c \cdot e^{\frac{1}{2} P(z)}+H(z), \quad c \in \mathrm{C},
$$

where

$$
H(z):=e^{\frac{1}{2} P(z)} \int_{a}^{z} S(t) e^{-\frac{1}{2} P(t)} d t, \quad a \in \mathrm{C} .
$$

By Lemma 2.2 and Lemma 2.3, it follows from (2.13) that $h^{\prime}(z)$ is of rational growth along $\Gamma$. On the other hand, by (2.5), we have

$$
|Q| \leqq\left|h^{\prime}\right|^{2}+\left|h^{\prime}\right| \cdot\left|\frac{h^{\prime \prime}}{h^{\prime}}\right|+2\left|h^{\prime}\right| \cdot\left|\frac{\pi^{\prime}}{\pi}\right|+\left|\frac{\pi^{\prime \prime}}{\pi}\right|+\left|e^{P}\right| .
$$

Since $h^{\prime}$ and $\pi$ are of finite order, Lemma 2.2, Lemma 2.4 and (2.14) imply that $Q(z)$ is of rational growth along $\Gamma$.

Since $\sigma(Q)<n$, we may apply the same Phragmén-Lindelöf type argument as we did above for $S(z)$ to show that $Q(z)$ is a polynomial, a contradiction. This completes the proof of Theorem 2:1.

Similar arguments in the corresponding higher order case result in

THEOREM 2.3. Consider the equation

$$
f^{(k)}+A_{k-2}(z) f^{(k-2)}+\ldots+A_{1}(z) f^{\prime}+\left(e^{P(z)}+A_{0}(z)\right) f=0,
$$

where $k \geqq 3, P(z)$ is a polynomial of degree $n \geqq 1$, and $A_{1}, A_{2}, \ldots, A_{k-2}$ are polynomials, while $A_{0}$ is a transcendental entire function of order $<n$. Then any solution $f \neq 0$ of (2.15) satisfies $\lambda(f)=\infty$.

We omit here the proof of Theorem 2.3. See also [7]. 
2.2. Proof of TheOrem 2.2. Let $f$ be a non-trivial solution to (2.1) such that $\lambda(f)<\infty$. We then prove that $f$ has no zeros and (2.2) holds. Since $\sigma(f)=\infty$, we may write $f=H e^{g}$, where $g$ is transcendental entire, and $H$ is the canonical product formed with the zeros of $f$, hence $\sigma(H)<\infty$. Substituting $f=H e^{g}$ into (2.1) we obtain

$$
-\left(g^{\prime}\right)^{2}=g^{\prime \prime}+2 \frac{H^{\prime}}{H} g^{\prime}+\frac{H^{\prime \prime}}{H}+A e^{B}+C .
$$

Since $B(z)$ is transcendental, a simple order consideration implies that $\sigma\left(g^{\prime}\right)=\infty$. Differentiating (2.16) yields

$$
-2 g^{\prime} g^{\prime \prime}=g^{\prime \prime \prime}+2\left(\frac{H^{\prime}}{H}\right)^{\prime} g^{\prime}+2 \frac{H^{\prime}}{H} g^{\prime \prime}+\left(\frac{H^{\prime \prime}}{H}\right)^{\prime}+\left(A^{\prime}+A B^{\prime}\right) e^{B}+C^{\prime} \text {. }
$$

Eliminating $e^{B}$ from (2.16) and (2.17) results in

$$
g^{\prime} R\left(g^{\prime}\right)=T\left(g^{\prime}\right),
$$

where $R\left(g^{\prime}\right), T\left(g^{\prime}\right)$ are two differential polynomials of $g^{\prime}$, with the following expressions:

$$
\begin{aligned}
& R\left(g^{\prime}\right):=2 A g^{\prime \prime}-\left(A^{\prime}+A B^{\prime}\right) g^{\prime} \\
& T\left(g^{\prime}\right):=-A g^{\prime \prime \prime}+\left(A^{\prime}+A B^{\prime}-2 A \frac{H^{\prime}}{H}\right) g^{\prime \prime} \\
&+2\left(\left(A^{\prime}+A B^{\prime}\right) \frac{H^{\prime}}{H}-A\left(\frac{H^{\prime}}{H}\right)^{\prime}\right) g^{\prime} \\
&+\left(A^{\prime}+A B^{\prime}\right) \frac{H^{\prime \prime}}{H}-A\left(\frac{H^{\prime \prime}}{H}\right)^{\prime}+\left(A^{\prime}+A B^{\prime}\right) C-A C^{\prime} .
\end{aligned}
$$

By Lemma 2.1, it follows from (2.18) that

$$
m(r, R) \leqq S\left(r, g^{\prime}\right)+O\left(m\left(r, B^{\prime}\right)+r^{\sigma_{1}}\right),
$$

where $\sigma_{1}<\infty$. On the other hand, we conclude by (2.16) that

$$
T\left(r, e^{B}\right)=m\left(r, e^{B}\right)=O\left(m\left(r, g^{\prime}\right)+r^{\sigma_{2}}\right), \quad r \notin \tilde{E}_{0},
$$

where $\sigma_{2}<\infty$ and $\widetilde{E}_{0}$ is a set in $[0, \infty)$ with finite linear measure. Hence,

$$
m\left(r, B^{\prime}\right)=m\left(r, \frac{\left(e^{B}\right)^{\prime}}{e^{B}}\right)=S\left(r, e^{B}\right)=o\left(m\left(r, g^{\prime}\right)+r^{\sigma_{2}}\right), \quad r \notin \tilde{E}_{0} .
$$

Combining (2.21) and (2.22) we obtain

$$
m(r, R)=S\left(r, g^{\prime}\right)+O\left(r^{\sigma_{3}}\right), \quad r \notin \tilde{E}_{0},
$$


for some $\sigma_{3}<\infty$. But now, substituting

$$
g^{\prime \prime}=\frac{1}{2 A}\left(A^{\prime}+A B^{\prime}\right) g^{\prime}+\frac{1}{2 A} R
$$

from (2.19) into (2.16) we get

$$
-2 A\left(g^{\prime}\right)^{2}-\left(A^{\prime}+A B^{\prime}+4 A \frac{H^{\prime}}{H}\right) g^{\prime}=2 A^{2} e^{B}+2 A \frac{H^{\prime \prime}}{H}+2 A C+R .
$$

Differentiating (2.25), eliminating $e^{B}$ similarly as was done for (2.18), and making use of (2.24), we obtain

$$
g^{\prime} V=W
$$

where

$$
\begin{gathered}
V=\frac{1}{2}\left(3 A^{\prime}+A B^{\prime}\right)\left(A^{\prime}+A B^{\prime}+4 A \frac{H^{\prime}}{H}\right) \\
-A\left(A^{\prime}+A B^{\prime}+4 A \frac{H^{\prime}}{H}\right)^{\prime}-2 A R, \\
W=A\left(2 A \frac{H^{\prime \prime}}{H}+2 A C+R\right)^{\prime}+\frac{1}{2} R\left(A^{\prime}+A B^{\prime}+4 A \frac{H^{\prime}}{H}\right) \\
-\left(2 A^{\prime}+A B^{\prime}\right)\left(2 A \frac{H^{\prime \prime}}{H}+2 A C+R\right) .
\end{gathered}
$$

Suppose now that $V$ in (2.27) does not vanish identically. From (2.26), we conclude that

$$
\begin{aligned}
m\left(r, g^{\prime}\right) & =T\left(r, g^{\prime}\right) \leqq T(r, W)+T(r, V)+O(1) \\
& \leqq O\left(m(r, R)+m\left(r, B^{\prime}\right)+r^{\sigma_{4}}\right) \leqq S\left(r, g^{\prime}\right)+O\left(r^{\sigma_{5}}\right)
\end{aligned}
$$

for some $\sigma_{4}<\infty, \sigma_{5}<\infty$, by (2.22) and (2.23). Therefore, $g^{\prime}$ is of finite order, contradicting the passage after (2.16). Hence, $V$ must vanish identically.

We now set

$$
2 H_{0}:=\frac{A^{\prime}}{A}+B^{\prime}, \quad u:=\frac{H^{\prime}}{H} .
$$

Making use of $u^{\prime}+u^{2}=H^{\prime \prime} / H$, and of (2.24), we may rewrite (2.27) in the form

$$
H_{0}^{2}-H_{0}^{\prime}+2 u H_{0}-2 u^{\prime}+2 g^{\prime} H_{0}-2 g^{\prime \prime}=0 .
$$

An elementary Laurent expansion consideration in (2.30) implies that $A$ and $H$ must be zero-free. Hence $f$ admits no zeros and we may assume $H=1$. 
Therefore, (2.27), (2.28) and (2.30) may be rewritten as

$$
\begin{aligned}
& R=A\left(H_{0}^{2}-H_{0}^{\prime}\right), \\
& 2 C^{\prime}-4 H_{0} C-H_{0}^{3}+3 H_{0} H_{0}^{\prime}-H_{0}^{\prime \prime}=0 .
\end{aligned}
$$

Now, (2.31) may be solved explicitly by

$$
C=c_{0} A e^{B}+\frac{1}{4}\left(\frac{A^{\prime}}{A}+B^{\prime}\right)^{\prime}-\frac{1}{16}\left(\frac{A^{\prime}}{A}+B^{\prime}\right)^{2}, \quad c_{0} \in \mathrm{C} .
$$

But now, we must have $c_{0}=0$ in (2.32), since $\sigma(C)<\infty, \sigma(A)<\infty, B$ is transcendental and $T\left(r, B^{\prime}\right)=S\left(r, e^{B}\right)$, see (2.22). Hence,

$$
C=-\frac{1}{16}\left(\frac{A^{\prime}}{A}+B^{\prime}\right)^{2}+\frac{1}{4}\left(\frac{A^{\prime}}{A}+B^{\prime}\right)^{\prime} .
$$

This completes the proof of Theorem 2.2.

\section{The higher order case.}

This section is devoted to giving, in the case of higher order linear differential equations, two results corresponding to those in Section 2. A partial counterpart to Theorem $\mathrm{A}$ is the following

THEOREM 3.1. Let $P$ be a polynomial of degree $n \geqq 1$, and let $Q_{0}, Q_{1}, \ldots, Q_{k-2}$, $k \geqq 3$, be entire functions of order $<n$. Suppose the linear differential equation

$$
f^{(k)}+Q_{k-2}(z) f^{(k-2)}+\ldots+Q_{1}(z) f^{\prime}+\left(e^{P(z)}+Q_{0}(z)\right) f=0
$$

admits a non-trivial solution $f$ such that $\lambda(f)<n$. Then $f$ has no zeros.

Proof. This proof is nothing but a higher order variant of the standard proof for Theorem A, see [14], Theorem 5.17. Therefore, we omit some details. On the other hand, due to complications arising from higher order terms, the assertion remains less precise than in the second order case.

Suppose now that (3.1) admits a non-trivial solution $f$ such that $\lambda(f)<n$. Write $f$ in the form $f=H e^{g}$, where $g$ is entire transcendental and $H$ is the canonical product formed with the zeros of $f$; hence $\sigma(H)<n$. Next, it is immediate by induction that

$$
\frac{f^{(k)}}{f}=\left(g^{\prime}\right)^{k}+\left(\begin{array}{l}
k \\
2
\end{array}\right)\left(g^{\prime}\right)^{k-2} g^{\prime \prime}+k \frac{H^{\prime}}{H}\left(g^{\prime}\right)^{k-1}+P_{k-2}\left(g^{\prime}\right)
$$

for all $k \geqq 1$. Here $P_{k-2}\left(g^{\prime}\right)$ stands for a differential polynomial in $g^{\prime}$ of total degree at most $(k-2)$ with coefficients which are polynomials in $H^{\prime} / H, H^{\prime \prime} / H, \ldots$, having constant coefficients. 
Substituting (3.2) into (3.1) results in

$$
-\left(g^{\prime}\right)^{k}=\left(\begin{array}{l}
k \\
2
\end{array}\right)\left(g^{\prime}\right)^{k-2} g^{\prime \prime}+k \frac{H^{\prime}}{H}\left(g^{\prime}\right)^{k-1}+P_{k-2}\left(g^{\prime}\right)+e^{P}+Q_{0},
$$

where the coefficients of $P_{k-2}\left(g^{\prime}\right)$ are now polynomials in $Q_{1}, \ldots, Q_{k-2}, H^{\prime} / H$, $H^{\prime \prime} / H, \ldots$, with constant coefficients. By Lemma 2.1, we infer $\sigma\left(g^{\prime}\right) \leqq n$ from (3.3). On the other hand, if $\sigma\left(g^{\prime}\right)<n$, estimating $m\left(r, e^{P}\right)$ by (3.3) results in an immediate contradiction. Hence $\sigma\left(g^{\prime}\right)=n$.

Differentiating now (3.3), and eliminating $e^{P}$ from (3.3) and the differentiated equation, yields

$$
\begin{aligned}
& k\left(g^{\prime \prime}-\frac{1}{k} P^{\prime} g^{\prime}\right)\left(g^{\prime}\right)^{k-1}=k\left(P^{\prime} \frac{H^{\prime}}{H}-\left(\frac{H^{\prime}}{H}\right)^{\prime}\right)\left(g^{\prime}\right)^{k-1} \\
& +\left(\begin{array}{l}
k \\
2
\end{array}\right)\left(g^{\prime}\right)^{k-3}\left(P^{\prime} g^{\prime} g^{\prime \prime}-(k-2)\left(g^{\prime \prime}\right)^{2}-g^{\prime} g^{\prime \prime \prime}\right) \\
& -k(k-1) \frac{H^{\prime}}{H}\left(g^{\prime}\right)^{k-2} g^{\prime \prime}+P_{k-2}\left(g^{\prime}\right) .
\end{aligned}
$$

Applying Lemma 2.1 once more, we see that

$$
g^{\prime \prime}-\frac{1}{k} P^{\prime}(z) g^{\prime}=S(z),
$$

where $\sigma(S)<n$. Moreover, we may use (3.5) to find expressions for $g^{\prime \prime}, g^{\prime \prime \prime}, \ldots$, in terms of $g^{\prime}$ and of $P^{\prime}, S$, and their derivatives. Substituting these expressions into (3.4) implies that

$$
\left(k S-\left(\begin{array}{l}
k \\
2
\end{array}\right) \frac{1}{k^{2}}\left(P^{\prime 2}-k P^{\prime \prime}\right)-P^{\prime} \frac{H^{\prime}}{H}+k\left(\frac{H^{\prime}}{H}\right)^{\prime}\right)\left(g^{\prime}\right)^{k-1}=P_{k-2}\left(g^{\prime}\right) .
$$

Denoting

$$
\alpha:=k S-\left(\begin{array}{l}
k \\
2
\end{array}\right) \frac{1}{k^{2}}\left(P^{\prime 2}-k P^{\prime \prime}\right)-P^{\prime} \frac{H^{\prime}}{H}+k\left(\frac{H^{\prime}}{H}\right)^{\prime},
$$

we conclude from (3.6) that

$$
\begin{aligned}
(k-1) m\left(r, g^{\prime}\right) & =m\left(r,\left(g^{\prime}\right)^{k-1}\right) \leqq m\left(r, \frac{1}{\alpha}\right)+m\left(r, P_{k-2}\left(g^{\prime}\right)\right) \\
& \leqq T(r, \alpha)+(k-2) m\left(r, g^{\prime}\right)+S\left(r, g^{\prime}\right)
\end{aligned}
$$

by writing $P_{k-2}\left(g^{\prime}\right)$ as a polynomial in $g^{\prime}$ whose coefficients are polynomials in $Q_{1}, \ldots, Q_{k-2}, H^{\prime} / H, H^{\prime \prime} / H, \ldots, g^{\prime \prime} / g^{\prime}, g^{\prime \prime \prime} / g^{\prime}, \ldots$, and applying a standard proxim- 
ity function estimate. This implies the contradiction $\sigma(\alpha) \geqq \sigma\left(g^{\prime}\right)$, unless

$$
k\left(\frac{H^{\prime}}{H}\right)^{\prime}-P^{\prime} \frac{H^{\prime}}{H}+k S-\left(\begin{array}{l}
k \\
2
\end{array}\right) \frac{1}{k^{2}}\left(P^{\prime 2}-k P^{\prime \prime}\right)=0 .
$$

Assuming that $H$ has a zero at $z_{0}$, an elementary pole order consideration in (3.7) implies a contradiction. Hence, $f$ cannot have zeros.

ReMARK. If $f$ has no zeros, we may assume $H=1$. Then $S=\left(\begin{array}{l}k \\ 2\end{array}\right) \frac{1}{k^{3}}$ $\left(P^{\prime 2}-k P^{\prime \prime}\right)$ by (3.7). The resulting equation for $g^{\prime}$, see (3.5), immediately implies

$$
g^{\prime}=C e^{\frac{1}{k} P}-\frac{k-1}{2 k} P^{\prime}, \quad C \neq 0 .
$$

This representation could be used to obtain expressions for $Q_{0}, \ldots, Q_{k-2}$ in terms of $P^{\prime}, P^{\prime \prime}, \ldots$ We omit such details except for the following related special case.

THEOREM 3.2. Let $P$ be non-constant entire, $Q_{0}, Q_{1}, Q_{2}$ be entire functions of order $<\sigma_{0}:=\sigma\left(e^{P}\right)$ and $Q_{1} \neq 0$. Suppose the linear differential equation

$$
f^{\prime \prime \prime}+Q_{2}(z) f^{\prime}+\left(Q_{1}(z) e^{P(z)}+Q_{0}(z)\right) f=0
$$

admits a non-trivial solution $f$ such that $\lambda(f)<\sigma\left(e^{P}\right)$. Then $f$ is zero-free, and

$$
\begin{aligned}
& Q_{0}=\frac{1}{3}\left(\frac{Q_{1}^{\prime}}{Q_{1}}+P^{\prime}\right)^{\prime \prime}-\frac{1}{9}\left(\frac{Q_{1}^{\prime}}{Q_{1}}+P^{\prime}\right)^{\prime}\left(\frac{Q_{1}^{\prime}}{Q_{1}}+P^{\prime}\right), \\
& Q_{2}=-\frac{1}{9}\left(\frac{Q_{1}^{\prime}}{Q_{1}}+P^{\prime}\right)^{2}+\frac{2}{3}\left(\frac{Q_{1}^{\prime}}{Q_{1}}+P^{\prime}\right)^{\prime} .
\end{aligned}
$$

Moreover, $Q_{1}$ also is zero-free.

PROOF. In a way similar to the proceeding argument we obtain

$$
\begin{aligned}
& -\left(g^{\prime}\right)^{3}=g^{\prime \prime \prime}+3 g^{\prime} g^{\prime \prime}+3 \frac{H^{\prime}}{H} g^{\prime \prime}+3 \frac{H^{\prime}}{H} g^{\prime 2} \\
& +\left(3 \frac{H^{\prime \prime}}{H}+Q_{2}\right) g^{\prime}+Q_{1} e^{P}+Q_{0}+\frac{H^{\prime}}{H} Q_{2}+\frac{H^{\prime \prime \prime}}{H},
\end{aligned}
$$

where $g$ and $H, \sigma(H)<\sigma_{0} \leqq \infty$ come from the standard Hadamard representation $f=H e^{g}$.

Again as in the preceding argument we obtain $\sigma\left(g^{\prime}\right)=\sigma_{0}$ by Clunie-type reasoning and by standard order estimates in (3.9). Defining $3 H_{0}:=\frac{Q_{1}^{\prime}}{Q_{1}}+P^{\prime}$, differentiating (3.9) and eliminating $e^{P}$ we get 


$$
\begin{aligned}
& \quad\left(g^{\prime \prime}-H_{0} g^{\prime}\right)\left(g^{\prime}\right)^{2}=-\frac{1}{3} g^{(4)}+\left(H_{0}-\frac{H^{\prime}}{H}\right) g^{\prime \prime \prime}-g^{\prime \prime \prime} g^{\prime}-\left(g^{\prime \prime}\right)^{2} \\
& +\left(3 H_{0}-2 \frac{H^{\prime}}{H}\right) g^{\prime \prime} g^{\prime}+\left(3 H_{0} \frac{H^{\prime}}{H}-\left(\frac{H^{\prime}}{H}\right)^{\prime}-\frac{H^{\prime \prime}}{H}-\frac{1}{3} Q_{2}\right) g^{\prime \prime} \\
& +\left(3 H_{0} \frac{H^{\prime}}{H}-\left(\frac{H^{\prime}}{H}\right)^{\prime}\right)\left(g^{\prime}\right)^{2}+\left(H_{0}\left(3 \frac{H^{\prime \prime}}{H}+Q_{2}\right)-\left(\frac{H^{\prime \prime}}{H}+\frac{1}{3} Q_{2}\right)^{\prime}\right) g^{\prime} \\
& +Q_{0} H_{0}+H_{0}\left(\frac{H^{\prime}}{H} Q_{2}+\frac{H^{\prime \prime \prime}}{H}\right)-\frac{1}{3} Q_{0}^{\prime}-\frac{1}{3}\left(\frac{H^{\prime}}{H} Q_{2}+\frac{H^{\prime \prime \prime}}{H}\right)^{\prime} .
\end{aligned}
$$

Applying Lemma 2.1 again, we see that

$$
g^{\prime \prime}-H_{0} g^{\prime}=S(z), \quad \text { where } \sigma(S)<\sigma_{0} .
$$

Differentiating (3.11) twice, and substituting the expressions for $g^{\prime \prime}, g^{\prime \prime \prime}, g^{(4)}$ into (3.10) we obtain

$$
A(z)\left(g^{\prime}\right)^{2}+B(z) g^{\prime}+C(z)=0
$$

where

$$
\begin{aligned}
A(z)=- & S-H_{0}^{\prime}+H_{0}^{2}+H_{0} \frac{H^{\prime}}{H}-\left(\frac{H^{\prime}}{H}\right)^{\prime}, \\
B(z)=- & \frac{1}{3}\left(H_{0}^{\prime \prime}+3 H_{0} H_{0}^{\prime}+H_{0}^{3}\right)+\left(H_{0}^{\prime}+H_{0}^{2}\right)\left(H_{0}-\frac{H^{\prime}}{H}\right)-\left(S^{\prime}+H_{0} S\right)-2 H_{0} S \\
& +S\left(3 H_{0}-2 \frac{H^{\prime}}{H}\right)+H_{0}\left(3 H_{0} \frac{H^{\prime}}{H}-\left(\frac{H^{\prime}}{H}\right)^{\prime}-\frac{H^{\prime \prime}}{H}-\frac{1}{3} Q_{2}\right) \\
& +H_{0}\left(3 \frac{H^{\prime \prime}}{H}+Q_{2}\right)-\left(\frac{H^{\prime \prime}}{H}+\frac{1}{3} Q_{2}\right)^{\prime},
\end{aligned}
$$

and

$$
\begin{aligned}
C(z)=- & \frac{1}{3}\left(S^{\prime \prime}+H_{0} S^{\prime}+\left(2 H_{0}^{\prime}+H_{0}^{2}\right) S\right)+\left(H_{0}-\frac{H^{\prime}}{H}\right)\left(S^{\prime}+H_{0} S\right)-S^{2} \\
& +\left(3 H_{0} \frac{H^{\prime}}{H}-\left(\frac{H^{\prime}}{H}\right)^{\prime}-\frac{H^{\prime \prime}}{H}-\frac{1}{3} Q_{2}\right) S+Q_{0} H_{0}+H_{0}\left(\frac{H^{\prime}}{H} Q_{2}+\frac{H^{\prime \prime \prime}}{H}\right) \\
& -\frac{1}{3} Q_{0}^{\prime}-\frac{1}{3}\left(\frac{H^{\prime}}{H} Q_{2}+\frac{H^{\prime \prime \prime}}{H}\right)^{\prime} .
\end{aligned}
$$

By (3.11) and standard estimates, $A, B, C$ are all of order $<\sigma\left(g^{\prime}\right)=\sigma_{0}$. By evaluating (3.12) at an appropriate sequence of points where the function $g^{\prime}$ assumes its maximum values, we obtain a contradiction, unless $A(z), B(z)$ and 
$C(z)$ all vanish identically. Combining the definitions of $S$ and $H_{0}$ with $A(z) \equiv 0$, it is easy to see by elementary Laurent expansion considerations that $H$, and hence $f$ must be non-vanishing entire functions. Thus, we may assume $H=1$, and the equations for $A, B, C$ reduce to

$$
\begin{gathered}
H_{0}^{2}-H_{0}^{\prime}=S, \\
-\frac{1}{3} H_{0}^{\prime \prime}+\frac{2}{3} H_{0}^{3}-S^{\prime}+\frac{2}{3} Q_{2} H_{0}-\frac{1}{3} Q_{2}^{\prime}=0, \\
-\frac{1}{3} S^{\prime \prime}+\frac{2}{3}\left(H_{0} S^{\prime}-H_{0}^{\prime} S+H_{0}^{2} S\right)-S^{2}-\frac{1}{3} Q_{2} S+Q_{0} H_{0}-\frac{1}{3} Q_{0}^{\prime}=0 .
\end{gathered}
$$

Substituting (3.13) into (3.14) results in

$$
Q_{2}^{\prime}-2 H_{0} Q_{2}-2 H_{0}^{3}+6 H_{0} H_{0}^{\prime}-2 H_{0}^{\prime \prime}=0,
$$

which can be solved explicitly. Hence, it is immediate, since $\sigma\left(Q_{2}\right)<\sigma_{0}$ and $m\left(r, H_{0}\right)=S\left(r, e^{P}\right)$, that

$$
Q_{2}=-H_{0}^{2}+2 H_{0}^{\prime}=-\frac{1}{9}\left(\frac{Q_{1}^{\prime}}{Q_{1}}+P^{\prime}\right)^{2}+\frac{2}{3}\left(\frac{Q_{1}^{\prime}}{Q_{1}}+P^{\prime}\right)^{\prime}
$$

is the only solution to (3.16) which is of order $<\sigma_{0}$. Similarly, substituting (3.13) and (3.17) into (3.15) yields

$$
Q_{0}^{\prime}-3 H_{0} Q_{0}=H_{0}^{\prime \prime \prime}+3 H_{0}^{2} H_{0}^{\prime}-H_{0}^{\prime 2}-4 H_{0} H_{0}^{\prime \prime} .
$$

Again, explicitly solving (3.18) we see that

$$
Q_{0}=\frac{1}{3}\left(\frac{Q_{1}^{\prime}}{Q_{1}}+P^{\prime}\right)^{\prime \prime}-\frac{1}{9}\left(\frac{Q_{1}^{\prime}}{Q_{1}}+P^{\prime}\right)^{\prime}\left(\frac{Q_{1}^{\prime}}{Q_{1}}+P^{\prime}\right)
$$

is the only solution to (3.18) of order $<\sigma_{0}$. Finally, (3.17) immediately implies that $Q_{1}$ has no zeros.

\section{The periodic case.}

In the periodic case, oscillation results usually rely on certain representations of solutions, see [3], Theorem 1 , for a prototype of such representations. If $f_{1}, f_{2}$ are two linearly independent solutions of $f^{\prime \prime}+A(z) f=0$ with a few zeros only, and $A(z)$ is rational in $e^{z}$, it was shown in [1], Lemma 3.1, that their corresponding representations are closely related. The proof of Theorem 4.1 applies a similar technique in the case where $A(z)$ is of finite order of growth and transcendental in $e^{z}$. In this case, the final conclusion $\max \left(\lambda\left(f_{1}\right), \lambda\left(f_{2}\right)\right)=\infty$ follows. Actually, Theorem 4.1 is nothing but a special case of [8], Theorem 4. However, we have included it here, since we feel that our proof may be modified to cover the case where $A(z)$ is of infinite order. Concerning some notation below, see [3]. 
THEOREM 4.1. Let $A(z)$ be a nonconstant entire function of period $2 \pi i$, of finite order of growth and transcendental in $e^{z}$. Let $f_{1}, f_{2}$ be two linearly independent solutions of $f^{\prime \prime}+A(z) f=0$. Then $\max \left(\lambda\left(f_{1}\right), \lambda\left(f_{2}\right)\right)=\infty$.

Proof. We first write $A(z)$ in the form $A(z)=B\left(e^{z}\right)$ where $B(\zeta)$ is transcendental and analytic on $0<|\zeta|<\infty$, and $\sigma_{0}(B)=\sigma_{\infty}(B)=0$. Supposing $\lambda\left(f_{1}\right)<\infty$, $\lambda\left(f_{2}\right)<\infty$, we may use [3], Theorem 4 and Theorem 5 , to obtain the representations

$$
f_{i}(z)=e^{d_{i} z} H_{i}\left(e^{z}\right) e^{g_{i}\left(e^{z}\right)}, \quad i=1,2,
$$

where $d_{i} \in \mathrm{C}, H_{i}(\zeta)$ and $g_{i}(\zeta)$ are analytic on $0<|\zeta|<\infty, \sigma_{0}\left(H_{i}\right)=\sigma_{\infty}\left(H_{i}\right)=0$ and $\sigma_{0}\left(g_{i}\right)=\sigma_{\infty}\left(g_{i}\right)=0$. Moreover, $g_{i}, i=1,2$, is of polynomial growth at $\zeta=0$, resp. $\zeta=\infty$, if and only if $B(\zeta)$ has at most a pole at $\zeta=0$, resp. $\zeta=\infty$. By [3], Lemma B,

$$
E(z)=f_{1}(z) f_{2}(z)=e^{\left(d_{1}+d_{2}\right) z} H_{1}\left(e^{z}\right) H_{2}\left(e^{z}\right) e^{g_{1}\left(e^{z}\right)+g_{2}\left(e^{z}\right)}
$$

is of finite order of growth. Therefore, $\exp \left(g_{1}\left(e^{z}\right)+g_{2}\left(e^{z}\right)\right)$ is of finite order, and so

$$
P(z):=g_{1}\left(e^{z}\right)+g_{2}\left(e^{z}\right)
$$

is a polynomial. Obviously, $P(z)$ is periodic by (4.2), and so it reduces to a complex constant, say $\beta$. Therefore,

$$
g_{2}\left(e^{z}\right)=-\mathrm{g}_{1}\left(\mathrm{e}^{z}\right)+\beta .
$$

Clearly, we may assume that $\beta=0$. By (4.3), we may rewrite (4.1) as

$$
f_{1}(z)=\phi_{1}(z) e^{G(z)}, \quad f_{2}(z)=\phi_{2}(z) e^{-G(z)},
$$

where

$$
\left\{\begin{array}{l}
\phi_{1}(z):=H_{1}\left(e^{z}\right), \\
\phi_{2}(z):=H_{2}\left(e^{z}\right) e^{\left(d_{1}+d_{2}\right) z}, \\
G(z):=g_{1}\left(e^{z}\right)+d_{1} z .
\end{array}\right.
$$

Denoting $n:=d_{1}+d_{2}$, we obtain by (4.5) that

$$
\left\{\begin{array}{l}
\frac{\phi_{1}^{\prime}(z)}{\phi_{1}(z)}=\frac{H_{1}^{\prime}\left(e^{z}\right)}{H_{1}\left(e^{z}\right)} e^{z} \\
\frac{\phi_{2}^{\prime}(z)}{\phi_{2}(z)}=n+\frac{H_{2}^{\prime}\left(e^{z}\right)}{H_{2}\left(e^{z}\right)} e^{z} \\
\phi_{1}(z) \phi_{2}(z)=H_{1}\left(e^{z}\right) H_{2}\left(e^{z}\right) e^{n z} \quad\left(=f_{1}(z) f_{2}(z)=E(z)\right), \\
G^{\prime}(z)=g_{1}^{\prime}\left(e^{z}\right) e^{z}+d_{1} .
\end{array}\right.
$$


Let $K \neq 0$ be the Wronskian of $f_{1}, f_{2}$. Then, by (4.4), (4.5) and (4.6),

$$
\begin{aligned}
K e^{-n z} \frac{1}{H_{1}\left(e^{z}\right) H_{2}\left(e^{z}\right)} & =\frac{K}{f_{1}(z) f_{2}(z)}=\frac{f_{2}^{\prime}(z)}{f_{2}(z)}=\frac{f_{1}^{\prime}(z)}{f_{1}(z)} \\
& =n-2 d_{1}+\frac{H_{2}^{\prime}\left(e^{z}\right)}{H_{2}\left(e^{z}\right)} e^{z}-\frac{H_{1}^{\prime}\left(e^{z}\right)}{H_{1}\left(e^{z}\right)} e^{z}-2 g_{1}^{\prime}\left(e^{z}\right) e^{z} .
\end{aligned}
$$

Hence we have in the $\zeta$-plane for $0<|\zeta|<\infty$ that

$$
K \zeta^{-n} \frac{1}{H_{1}(\zeta) H_{2}(\zeta)}=n-2 d_{1}+\zeta \frac{H_{2}^{\prime}(\zeta)}{H_{2}(\zeta)}-\zeta \frac{H_{1}^{\prime}(\zeta)}{H_{1}(\zeta)}-2 \zeta g_{1}^{\prime}(\zeta)
$$

To proceed now, we recall the Valiron representations $H_{i}(\zeta)=\zeta^{k_{1}} \Psi_{i}(\zeta) u_{i}(\zeta)$, $i=1,2$, where $k_{i} \in Z, \Psi_{i}$ is analytic in $0<|\zeta| \leqq \infty, \Psi_{i}(\infty) \neq 0$, and $u_{i}$ is entire and of order zero, see [14], Lemma 5.18. Similarly, $H(\zeta):=H_{1}(\zeta) H_{2}(\zeta)=\zeta^{k} \Psi(\zeta) u(\zeta)$. Moreover, for an entire function $v$ of order zero, we may apply the theorem on $\mathrm{p}$. 294 in [9] to conclude that

$$
\underline{\log } \underline{\text { dens }}\left\{r \geqq 0 \mid \log \mu(r, v)>\frac{7}{8} \log M(r, v)\right\} \geqq \frac{1}{2} .
$$

Here $\mu(r, v):=\min |v(z)|$. Hence,

$$
|z|=r
$$

$$
\mu(r, v)>\sqrt{M(r, v)} \geqq 1
$$

holds in a set of $r$-values of infinite logarithmic measure. Finally, the standard Wiman-Valiron reasoning applies to (4.7), since by the Valiron representation, one concludes that

$$
\frac{H_{i}^{\prime}(\zeta)}{H_{i}(\zeta)}=\frac{k_{i}}{\zeta}+\frac{\Psi_{i}^{\prime}(\zeta)}{\Psi_{i}(\zeta)}+\frac{u_{i}^{\prime}(\zeta)}{u_{i}(\zeta)}=\frac{u_{i}^{\prime}(\zeta)}{u_{i}(\zeta)}+O(1)
$$

Let $\zeta$ be such that (4.8) applies for $|\zeta|=r$ as well as for the Wiman-Valiron theorem, see [14], Theorem 3.2, relative to $u_{1}$ and $u_{2}$. Then, by (4.7), (4.8), [14], Proposition 3.3, and the fact that the fact that the entire functions $u, u_{1}, u_{2}$ are of order zero, we get

$$
\begin{aligned}
2 r\left|g_{1}^{\prime}(\zeta)\right| & \leqq O(1)+v\left(r, u_{1}\right)+v\left(r, u_{2}\right)+O(r)+O\left(r^{-n-k} \frac{1}{|u(\zeta)|}\right) \\
& \leqq O\left(r^{-n-k} \frac{1}{\mu(r, u)}\right)+O(r)=O(r)
\end{aligned}
$$

as $r \rightarrow \infty$ for all such $\zeta$. Here $v\left(r, u_{i}\right)$ is the central index for $u_{i}, i=1,2$. Since $\sigma_{\infty}\left(g_{1}^{\prime}\right)=0, g_{1}^{\prime}$ must be of polynomial growth at $\zeta=\infty$. The point $\zeta=0$ can be treated similarly, hence $g_{1}(\zeta)$, and so $B(\zeta)$ also, must be a rational function, a contradiction. 
REMARK. The assumption in Theorem 4.1 that the order of $A(z)$ is finite is necessary. In fact, the differential equation

$$
f^{\prime \prime}+\left(e^{e^{z}}-\frac{1}{16} e^{2 z}+\frac{1}{4} e^{z}\right) f=0
$$

admits two linearly independent zero-free solutions, namely

$$
f(z)=\exp \left(-\frac{1}{2}\left(\frac{1}{2} e^{z} \pm 2 i \int_{0}^{z} e^{\frac{1}{2} e^{t}} d t\right)\right) .
$$

\section{REFERENCES}

1. S. Bank, A note on the oscillation of solutions of periodic linear differential equations, Czech. Math. J. 44 (1994), 91-107.

2. S. Bank and I. Laine, On the oscillation theory of $f^{\prime \prime}+A f=0$ where $A$ is entire, Trans. Amer. Math. Soc. 273 (1982), 351-363.

3. S. Bank and I. Laine, Representations of solutions of periodic second order linear differential equations, J. Reine Angew. Math. 344 (1983), 1-21.

4. S. Bank, I. Laine and J. Langley, On the frequency of zeros of solutions of second-order linear differential equations, Resultate Math. 10 (1986), 8-24.

5. S. Bank, I. Laine and J. Langley, Oscillation results for solutions of linear differential equations in the complex domain, Resultate Math. 16 (1989), 3-15.

6. S. Bank, and J. Langley, On the oscillation of solutions of certain linear differential equations in the complex domain, Proc. Edinburgh Math. Soc. 30 (1987), 455-469.

7. S. Bank, and J. Langley, On the zeros of the solutions of the equation $w^{(k)}+\left(R e^{P}+Q\right) w=0$, Kodai Math. J. 13 (1990), 298-309.

8. S. Bank, and J. Langley, Oscillation theorems for higher order linear differential equations with entire periodic coefficients, Comment. Math. Univ. St. Paul. 41 (1992), 65-85.

9. P. D. Barry, On a theorem of Besicovitch, Quart. J. Math. Oxford (2) 14 (1963), 293-302.

10. J. Clunie, On integral and meromorphic functions, J. London Math. Soc. 37 (1962), 17-27.

11. G. Gundersen, Estimates for the logarithmic derivative of a meromorphic function, plus similar estimates, J. London Math. Soc. 37 (1988), 88-104.

12. W. K. Hayman, Meromorphic Functions, Clarendon Press, Oxford, 1964.

13. G. Jank and L. Volkmann, Einführung in die Theorie der ganzen und meromorphen Funktionen mit Anwendungen auf Differentialgleichungen, Birkhäuser, Basel-Boston, 1985.

14. I. Laine, Nevanlinna Theory and Complex Differential Equations, W. de Gruyter, Berlin, 1993.

15. J. K. Langley, On complex oscillation and a problem of Ozawa, Kodai Math. J. 9 (1986), 430-439.

16. Shupei Wang, On the sectorial oscillation theory of $f^{\prime \prime}+A(z) f=0$, Ann. Acad. Sci. Fenn. Ser. A I Math. Diss. 92 (1994).

Y. M. CHIANG

DEPARTMENT OF MATHEMATICS

THE HONG KONG UNIVERSITY OF SCIENCE AND TECHNOLOGY

CLEAR WATER BAY

KOWLOON

HONG KONG
I. LAINE AND S. WANG DEPARTMENT OF MATHEMATICS UNIVERSITY OF JOENSUU

P.O. BOX 111

FIN-80101 JOENSUU

FINLAND 